\title{
Quantum-Field-Theoretic Simulation Platform for Observing the Fate of the False Vacuum
}

\author{
Steven Abel ${ }^{*}$ and Michael Spannowsky ${ }^{\dagger}$ \\ Department of Physics, Institute for Particle Physics Phenomenology, Durham University, Durham DH1 3LE, \\ United Kingdom
}

(Received 8 July 2020; accepted 22 January 2021; published 24 March 2021)

\begin{abstract}
We design and implement a quantum annealing simulation platform to observe and study dynamical processes in quantum field theory (QFT). Our approach encodes the field theory as an Ising model, which is then solved by a quantum annealer. As a proof of concept, we encode a scalar field theory and measure the probability for it to tunnel from the false vacuum to the true vacuum for various tunneling times, vacuum displacements, and potential profiles. The results are in accord with those predicted theoretically, showing that a quantum annealer is a promising platform for encoding QFTs. This is the first time it has been possible to measure instanton processes across a freely chosen QFT energy barrier. We argue that this novel and flexible method to study the dynamics of quantum systems has potential application to many field theories of interest. Measurements of the dynamical behavior of such encoded field theories are independent of theoretical calculations and can be used to infer their properties without being limited by the availability of suitable perturbative or nonperturbative computational methods. Soon, measurements using such a quantum annealing simulation platform could therefore be used to improve theoretical and computational methods conceptually and may enable the measurement and detailed study of previously unobserved quantum phenomena.
\end{abstract}

DOI: 10.1103/PRXQuantum.2.010349

\section{INTRODUCTION}

Quantum field theories (QFTs) are the theoretical framework underlying the most fundamental description of nature. Yet, studying the dynamics of those special classes of quantum field theories that occur in nature requires either the use of multibody quantum systems (e.g., condensed matter systems) or the design of highly sophisticated high-energy experiments that probe the properties of quantum field theories when they manifest themselves as particles. So far no quantum platform has been devised that provides a platform for studying and measuring the quantum effects and dynamics of arbitrary field theories; that is, theories in which the quantum numbers and interactions of quantum fields can be adjusted at will. We show that a quantum annealer acting on a generalized Ising model [1] can serve exactly that purpose - namely, it can be a simulation platform for field

\footnotetext{
*s.a.abel@durham.ac.uk

†michael.spannowsky@durham.ac.uk
}

Published by the American Physical Society under the terms of the Creative Commons Attribution 4.0 International license. Further distribution of this work must maintain attribution to the author(s) and the published article's title, journal citation, and DOI. theories chosen at will. Consequently, in soon many theoretical calculations for quantum field theories could be replaced by quantum measurements, thereby overcoming computational or theoretical limitations (e.g., perturbative or nonperturbative computational methods) or high computational demands (e.g., in lattice calculations). We refer to this as a "quantum annealing simulation platform" (QASP) (although some of the techniques we discuss for encoding a field theory would be applicable to quantum gate devices as well). Our study is performed on the DWave Systems quantum annealer, which we treat as a trusted device that does what it claims to do-namely, it implements the potential and interactions of a generalized Ising model. Under this assumption, our goal is to then test if the QASP produces results that fit with expectations.

We use the method introduced in Ref. [2] for encoding a field theory in a generalized Ising model, and show that, in principle, implemented on an annealer it allows one to implement and observe truly quantum dynamical processes. We focus on recreating and measuring the phenomenon of tunneling in scalar field theories, which is clear evidence for a quantum rather than a classical process. For a field theory that has $d=0$ or $d=1$ space-time dimensions, this is equivalent to the measurement of the time-dependent quantum mechanical wave function as it attempts to reach the ground state of the system. From a 
more general field theory perspective, it means that we are able to measure nonperturbative decay processes that are described by instantons [3-9].

In Yang-Mills theories [10] such objects are of profound importance, because they are the primary explicit example of genuinely nonperturbative gauge field configurations, leading to a wealth of geometrical, topological, and quantum effects with a fundamental impact on quantum dynamics. Instanton effects are important in the electroweak sector of the Standard Model and also in QCD, as well as in a broad variety of theoretical constructions ranging from supersymmetric models, grand unified theories, and extra dimensions to string theory and D-branes [11-16]. While all these incarnations of instantons have been predicted, and there is little doubt of their existence in the Standard Model and their profound role in shaping the history of the early universe, none of the relevant processes have been observed experimentally [17-21]. Reproducing and measuring such specific processes of interest as genuine quantum effects (rather than merely studying them numerically) has become an important goal (for complementary approaches see, e.g., Refs. [22-25]).

There are by contrast several specific condensed matter systems where such effects have been observed [26]. However, all these cases were constrained to the particular field theory in question. The ultimate ambition of this approach is to instead provide a framework for studying nonperturbative effects in any field theory of interest.

This would, in principle, allow one to check the calculation of nonperturbative phenomena by measuring them on a QASP. It may even be possible to observe new phenomena that have not yet been anticipated. For this study we are, of course, be limited by the hardware that is available to us, so the discussion is necessarily restricted to the simpler field theories that can exhibit instantonlike behavior - namely, the aforementioned $d=1$ scalar field theory. Nevertheless, within this theory we are be able to set up a potential that we then manipulate by hand so that it develops a nontrivial vacuum structure that induces tunneling. We believe this is the first time that it has been possible to implement instanton processes in a freely chosen quantum field theory and observe the expected phenomena.

\section{SETUP FOR FALSE-VACUUM DECAY}

It is convenient for several practical reasons to set up a physical system on the annealer that recreates quantum decay in a potential of the form

$$
U(\phi)=\frac{3}{4} \tanh ^{2} \phi-k(t) \operatorname{sech}^{2}[c(\phi-v)]
$$

where $c$ and $v$ are constants, while $k$ is time dependent, and $\phi(t)$ is the field. $\phi$ is the dimensionless object that we define on the annealer. When required we convert it into a

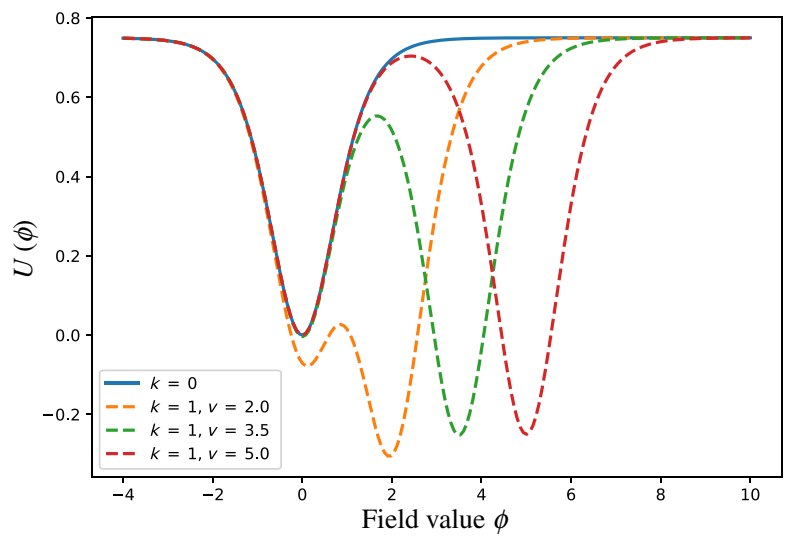

FIG. 1. The double Pöschl-Teller potential well for different values of $k$ and $v$. The system is initialized around $\phi=0$ and allowed to decay to the true minimum at $\phi \approx v$.

dimensionful field $\eta$ by defining

$$
\phi=\frac{\eta}{\eta_{0}}
$$

where $\eta_{0}$ is a constant. In the $d=1$ field theory there are, of course, no space dimensions, and at leading order it is isomorphic to quantum mechanics (with $\phi$ playing the role of $x$ ). However, the $d=1$ field theory formalism allows for particle creation and is the starting point for generalization to higher dimensions, as discussed in Sec. I.

The first term in $U$ provides a potential well around $\phi=0$, which, in principle, allows the system to begin as a bound state there. As mentioned, this is one of the benefits of annealers over discrete gate systems: in order first to reach a ground state, a system has to dissipate energy. Indeed, in the annealing the field is chosen to start at a fixed value, which implies that it is initially a $\delta$ function, effectively containing an equal measure of all energy eigenstates. At first, therefore, it has to shed energy to arrive at a ground state.

The $k$ term is then be turned on adiabatically during the annealing in order to allow tunneling into the global minimum that forms at $\phi=v$. For this study we mostly take $c=1$, so that the potential during the tunneling period consists of equally sized potential wells. The potential is plotted in Fig. 1 for $k=1$ and various values of separation parameter $v$.

This function has several nice properties for our purposes. One is that each individual well has the PöschlTeller $-\operatorname{sech}^{2} \phi$ form, which can be solved [27,28]. Moreover the potentials around each minimum decay exponentially. This makes it possible to "turn on" the global true minimum by adjusting $k$ without significantly altering the profile of the potential around the false minimum (unlike the more commonly considered case of quartic potentials). Other useful features of this choice are discussed below when they become relevant. 
We begin the system with $k=0$, such that it falls into a Pöschl-Teller ground state. Assuming that the completion of the potential into a $d=1$ field theory ultimately corresponds to the Schrödinger equation, the ground state (and its excited "friends") in such a potential can be determined by factorization and ladder-operator methods (see, e.g., Refs. [28,29]). In a theory where

$$
\frac{2 m \eta_{0}^{2}}{\hbar^{2}} U=\lambda(\lambda+1) \tanh ^{2} \phi,
$$

the bound states are given by Legendre polynomials of the form $P_{\lambda}^{\mu}(\tanh \phi)$, and the ground state, $P_{\lambda}^{\lambda}(\tanh \phi)$, is given by

$$
\psi_{0}(\phi)=\mathcal{N}_{0} \operatorname{sech}^{\lambda} \phi
$$

where the normalization constant is

$$
\mathcal{N}_{0}^{2}=\frac{\pi^{-1 / 2} \Gamma(\lambda+1 / 2)}{\Gamma(\lambda)} .
$$

This state, which is our idealized starting state, has energy

$$
E_{0}=\frac{\hbar^{2} \lambda}{2 m \eta_{0}^{2}} .
$$

We will not know a priori the value of

$$
\gamma \stackrel{\text { def }}{=} \frac{\hbar^{2}}{2 m \eta_{0}^{2}}
$$

in the effective field theory induced on the annealer, and estimating it will essentially constitute our calibration. To do this we could, for example, multiply $U$ by a constant, $\alpha$ say, and by trial and error find a value for $\alpha$ that yields a ground-state wave function of the form $\psi_{0}=\operatorname{sech} \phi / \sqrt{\pi}$ corresponding to $\lambda=1 / 2$. According to Eq. (3) that value of $\alpha$ would be equal to $\gamma$. However, this is demanding to do (in terms of annealing time), and it is not easy to determine the value of $\lambda$. We instead determine an estimate for $\gamma$ in the effective field theory by studying the ground state of the simple-harmonic-oscillator potential and fitting the wave function to the ground state. Either way it is unavoidable that one must also determine $\gamma$ as an empirical parameter.

We now consider the tunneling into the global minimum once $k$ is turned on. The expected decay rate can be computed by instanton methods. In $d=1$ field theory, this means writing the path integral for the nonrelativistic propagation of the physical field $\eta=\eta_{0} \phi$ as a worldline integral:

$$
\left\langle\eta_{i} \mid \eta_{f}\right\rangle=\int_{\eta(0)=\eta_{i}}^{\eta(T)=\eta_{f}} \mathcal{D} \eta e^{-i \hbar^{-1} \int_{0}^{T} d t\left[\frac{1}{2} m \dot{\eta}^{2}-\left(U-E_{0}\right)\right]},
$$

where the path is between points $\eta_{i}$ inside and $\eta_{f}$ outside the barrier, and $T$ is the time. As usual the integral is dominated by the stationary phase contribution, but to evaluate it efficiently, we deform $t$ in the complex $t$ plane by making a Wick rotation $t \rightarrow-i t$ and use the Euclidean steepest-descent contour instead:

$$
\left\langle\eta_{i} \mid \eta_{f}\right\rangle_{E}=\int_{\eta(0)=\eta_{i}}^{\eta(T)=\eta_{f}} \mathcal{D} \eta e^{-\hbar^{-1} \int d t\left(m \dot{\eta}^{2} / 2+U-E_{0}\right)} .
$$

This describes the propagator from $\eta_{i}$ to the endpoint, but we are most interested in the exponentially decaying part. The steepest-descent contour that determines it corresponds to the classical solution of the Euclidean equation of motion $\eta_{\mathrm{cl}}$ with endpoints at $\eta_{+}$and $\eta_{e}$, where $\eta_{e}$ is the escape point, namely, the point where $U=E_{0}$, with the quantum fluctuations providing prefactors. That is,

$$
\delta S_{E}=0 \Longrightarrow m \ddot{\eta}=U_{\eta},
$$

which gives the usual classical solution

$$
\dot{\eta}_{\mathrm{cl}}= \pm \sqrt{\frac{2\left(U-E_{0}\right)}{m}}
$$

corresponding to energy conservation for a ball rolling in the inverted potential between turning points at $\eta_{+}$and $\eta_{e}$. Substitution then gives the classical action

$$
S_{E, \mathrm{cl}}=\int_{\eta_{+}}^{\eta_{e}} d \eta \sqrt{2 m\left(U-E_{0}\right)},
$$

and our letting $\eta=\eta_{\mathrm{cl}}+\delta \eta$ yields a quantum prefactor:

$$
\begin{aligned}
\left\langle\eta_{i} \mid \eta_{f}\right\rangle_{E} & =\int \mathcal{D} \delta \eta e^{-\hbar^{-1} \int d t\left[m\left(\dot{\eta}_{\mathrm{cl}}+\delta \dot{\eta}\right)^{2} / 2+U\left(\eta_{\mathrm{cl}}+\delta \eta\right)-E_{0}\right]} \\
& =A e^{-\hbar^{-1} S_{E, \mathrm{cl}}}
\end{aligned}
$$

with the decay rate $\Gamma=\left|\left\langle\eta_{i} \mid \eta_{f}\right\rangle_{E}\right|^{2}$ becoming

$$
\Gamma \approx e^{-2 \hbar^{-1} S_{E, \mathrm{cl}}} .
$$

In principle these solutions should then be matched to oscillating solutions at the turning points, but these oscillating parts do not change the decay rate. Thus regardless of the time $T$, the exponential decay in the amplitude between points on either side of the barrier will be dominated by this saddle point approximation, as one would expect. As mentioned, the $d=1$ field theory is isomorphic to the Schrödinger equation at leading order, and indeed the same result can be obtained by the WKB method.

How can we test this decay rate in a quantum annealer directly? The assumption we make is that the transverse field component of the annealer induces an effective $\dot{\phi}^{2}$ term into any field theory we encode on it, with some unknown coefficient. Therefore our method is to construct 


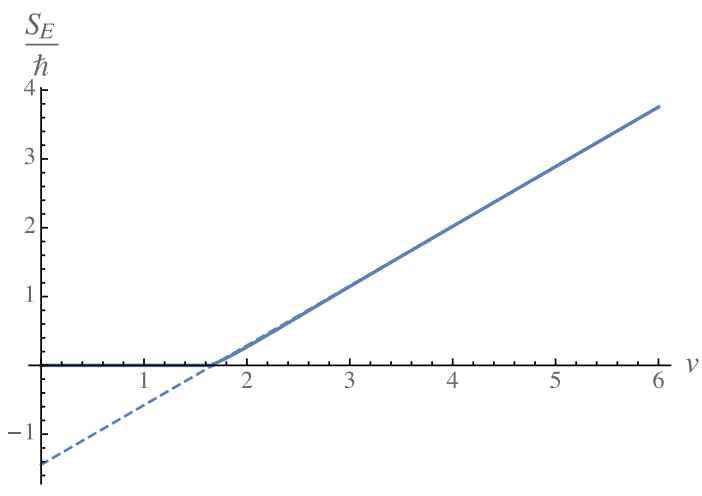

FIG. 2. The logarithm of the decay rate $\Gamma$ (multiplied by $-1 / 2$ ) versus the linear approximation in Eq. (14) (shown as the dashed line) for $\gamma=1$. The barrier disappears completely at $v=5 / 3$.

on the annealer a potential $U$ as given in Eq. (1) and, by observing its decay rates, test to see if the annealer has indeed turned it into a $d=1$ quantum field theory. The object of interest is therefore the exponent in the decay rate:

$$
\begin{aligned}
\hbar^{-1} S_{E} & =\int_{\eta_{+}}^{\eta e} \sqrt{\frac{2 m\left(U-E_{0}\right)}{\hbar^{2}}} d \eta \\
& \approx \gamma^{-1 / 2} \int_{\phi_{+}}^{\phi_{e}} \sqrt{\frac{3}{4} \tanh ^{2} \phi-\operatorname{sech}^{2}(\phi-v)} d \phi
\end{aligned}
$$

where we have set $c=1$. Obviously this integral becomes linear in $v$ at large values, but a second advantage of the Pöschl-Teller potential barrier is that it remains so to a very good approximation, even for values of $v$ of order 1 , as shown in Fig. 2:

$$
\log \Gamma \approx-2 \hbar^{-1} S_{E} \approx \sqrt{\frac{3}{\gamma}}\left(\frac{5}{3}-v\right) .
$$

Thus we expect exponential decay with an exponent decreasing linearly with $v$. Crucially this behavior is qualitatively different from thermal tunneling, which has little dependence on the barrier width $v$. For that one would instead expect to recover the Arrhenius equation, with $\Gamma \sim e^{-E_{a} / k T}$, where $E_{a}$ is the activation energy [30].

\section{IMPLEMENTATION ON A QUANTUM ANNEALER}

We now put together the components required to perform such a study. As mentioned, our goals are to encode the field theory potential $U(\phi)$ on the annealer, then put the system into the approximate ground state of a stable minimum, and add instability by adjusting the coupling $k$ in Eq. (1).
The method for encoding field theory is discussed in Ref. [2]. In short, we begin with the effective Hamiltonian of the annealer, which is a generalized Ising model of the form

$$
\begin{aligned}
\mathcal{H}_{\mathrm{QA}}= & B(s)\left(\sum_{i j} \hat{J}_{i j} \sigma_{i}^{Z} \sigma_{j}^{Z}+C(t) \sum_{i} \hat{h}_{i} \sigma_{i}^{Z}\right) \\
& +A(s) \sum_{i} \sigma_{i}^{X},
\end{aligned}
$$

where $i$ and $j$ label the qubits, $\sigma_{i}^{Z}$ are the $z$-spin Pauli matrices, and $\sigma_{i}^{X}$ are the transverse field components, while the couplings $\hat{h}_{i}$ and $\hat{J}_{i j}$ between the qubits are set and kept constant.

The reason these symbols are hatted is that they are not in general the ones $h_{i}$ and $J_{i j}$ that are input by the user. The annealer autoscales the latter until the largest absolute value of the coupling $h_{i}\left(J_{i j}\right)$ is $2(1)$. That is,

$$
\hat{h}_{i}=\frac{h_{i}}{\max \left\{\left|h_{i}\right| / 2,\left|J_{i j}\right|\right\}} ; \quad \hat{J}_{i j}=\frac{J_{i j}}{\max \left\{\left|h_{i}\right| / 2,\left|J_{i j}\right|\right\}} .
$$

In our study we keep all the couplings sufficiently small so that autoscaling is avoided (it is possible to extend the ranges of couplings but we do not do this here).

The parameter $s(t)$ (with $t$ being time) is a user-defined control parameter that can be adjusted during the annealing, while $A$ and $B$ describe the resulting change in the quantum characteristics of the annealer, and $C(t)$ is another user-defined parameter, called the " $h$ gain." To perform the more standard task of finding a global optimization, one would encode the problem to be solved in the "classical" Ising model represented by the $A$ terms, and then adjust the relative parameters $A$ and $B$ to perform an annealing from a highly quantum system to a classical one that has $B=0$. For our purposes we instead probe the quantum properties of the system when $B \neq 0$.

Scalar field values can be represented with the "domainwall encoding" introduced in Ref. [31]. That is, we first add the Ising chain Hamiltonian: if we define the total number of qubits we use as $N$ (where $N$ should be large), this is given by

$$
\begin{aligned}
& J_{i j}^{\text {(chain) }}=-\frac{\Lambda}{2}\left(\begin{array}{rrrrrr}
0 & 1 & & & & \\
1 & 0 & 1 & & & \\
& 1 & 0 & & & \\
& & & \ddots & & \\
& & & 0 & 1 \\
& & & 1 & 0
\end{array}\right), \\
& h^{\text {(chain) }}=\Lambda^{\prime}(1,0,0 \ldots, 0,-1),
\end{aligned}
$$

where $\Lambda$ and $\Lambda^{\prime}$ are parameters that are somewhat larger than the largest energy scale in the problem (for the best 
performance they should not be very much larger). The coupling $h^{\text {(chain) }}$ forces the system to have spin $\sigma_{1}^{Z}=-1$ at one end and spin $\sigma_{N}^{Z}=+1$ at the other, while $J^{\text {(chain) }}$ forces it to have as few spin flips as possible. The result is a single "frustrated" position (the so-called domain wall) where the spin flips from negative to positive. This position, $r$ say, encodes the value of the scalar field as

$$
\phi=\phi_{0}+\xi r=\phi_{0}+\frac{\xi}{2} \sum_{i=1}^{N}\left(1-\sigma_{i}\right)
$$

where $\phi_{0}$ is a fiducial minimum value, while the second term gives $r$ contributions of $\xi$ from the negative $\sigma_{i}^{Z}$ up to the domain-wall position. It is then straightforward to see that one can encode a potential term $U_{1}(\phi)$ in the $h_{i}$ couplings by adding

$$
h_{j}^{(\mathrm{QFT})}=-\frac{\xi}{2} U_{1}^{\prime}\left(\phi_{0}+\xi j\right) .
$$

For our purposes, such a term cannot represent the whole of $U$ in Eq. (1) however, because we need to divide the potential into two pieces to have the ability to turn on the metastable component. This functionality is provided by the $h$-gain parameter $C(t)$, so the entire potential is encoded as

$$
U=U_{0}+U_{1}
$$

where

$$
U_{0}=\frac{3}{4} \tanh ^{2} \phi, \quad U_{1}=-k(t) \operatorname{sech}^{2}(\phi-v),
$$

where $U_{0}$ remains to be encoded in $J$. This allows us first to allow the system to settle in the minimum around $\phi=0$ and then to adjust $C$ during the annealing to turn on the potential $U_{1}$ and induce tunneling. The encoding of $U_{0}$ into $J$ can be done by adding the couplings

$$
J_{i j}^{(\mathrm{QFT})}=\frac{1}{4} U_{0}\left(\phi_{0}+\xi j\right)\left(2 \delta_{i j}-\delta_{i(j-1)}-\delta_{(i-1) j}\right),
$$

where $\delta_{i j}$ is the Kronecker $\delta$. These $J$ terms contribute zero to the Hamiltonian except at the location of the domain wall, where $\left(2 \sigma_{k}^{Z} \sigma_{k}^{Z}-\sigma_{k}^{Z} \sigma_{k+1}^{Z}-\sigma_{k+1}^{Z} \sigma_{k}^{Z}\right)=4$, yielding a contribution $U_{0}(\phi)$ at that point.

$h^{\text {(chain) }}$ is also scaled down when $C(t)$ is small, so with this simple encoding we cannot set $C=0$. However, we do not need to initially turn off $U_{1}$ entirely, but we just need to reduce it so that tunneling is not possible. A more precise encoding that allows one to turn off $U_{1}$ entirely is to share $U_{1}$ between $J$ and $h$ such that the initial value of $C$ makes

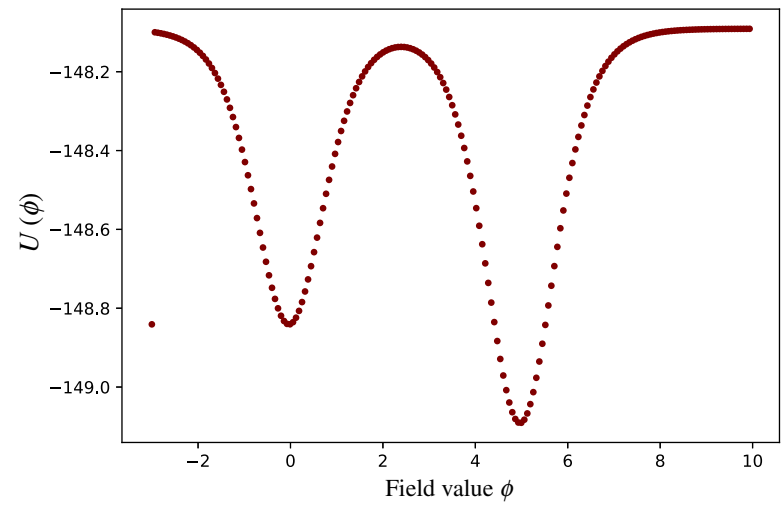

FIG. 3. The potential as seen by the Ising model on the annealer, where we choose $N=200$ qubits and $k=1$ and $v=5$; cf. the actual potential in Fig. 1. Note the large negative overall energy offset due to the field theory encoding, and the "dropped qubit" at $\phi=\phi_{0}$.

them cancel exactly. That is,

$$
\begin{aligned}
J_{i j}^{(U)}= & \frac{1}{4}\left(U_{0}\left(x_{0}+\xi j\right)-\frac{C_{0}}{1-C_{0}} U_{1}\left(x_{0}+\xi j\right)\right) \\
& \left(2 \delta_{i j}-\delta_{i(j-1)}-\delta_{(i-1) j}\right), \\
h_{j}^{(U)}= & -\frac{\xi}{2} \frac{1}{1-C_{0}} U_{1}^{\prime}\left(x_{0}+\xi j\right),
\end{aligned}
$$

where the choice of parameters $C(0)=C_{0}$ and $C\left(t_{f}\right)=1$ gives the desired behavior. We use this later, but for the moment we stay with the simpler assignment of potentials.

This completes the encoding of the field theory potential. To verify that it is working as desired, we show the resulting potential in Fig. 3. For this and the remainder of the work we take $N=200$ as a reasonable compromise between accuracy and efficiency on the annealer. As expected there are two unavoidable features of the Ising potential compared with the original one, both caused by the Ising chain encoding of the field theory: first, the negative rewards in $J^{\text {(chain) }}$ cause an offset of order $-N \Lambda$; second, the rewards in $h^{\text {(chain) }}$ in Eq. (17) imply "dropped qubits" at the first and last positions (the one at the last position is off the scale). Neither of these should affect the tunneling rate.

We now turn to the configuration of the annealing itself. As mentioned, the coefficients $A$ and $B$ describe how "quantum" the system is, and are best visualized with the plot in Fig. 4. When $s=0$, the system is maximally quantum, and when $s=1$, the system has arrived at the pure classically Ising-encoded problem. A "forward annealing" schedule would take $s\left(t_{i}\right)=0$ and $s\left(t_{f}\right)=1$, beginning with a rapidly tunneling system and ending up with a system that solves the optimization problem of interest. A "reverse annealing" schedule gains initial classical control with $s=1$. Then we turn on the quantum mechanics so 


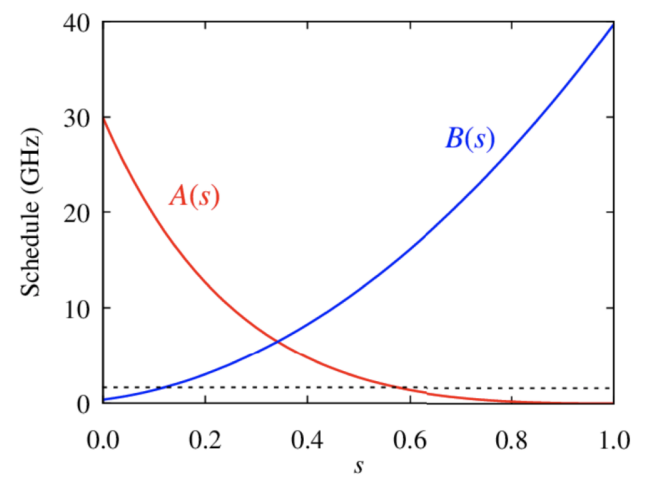

FIG. 4. Annealing schedule parameters. The thermal contribution is shown as a solid line, while $B$ and $A$ are the coefficients scaling the classical Ising and transverse field contributions, respectively.

that we send $s$ to some finite value $s_{q}$ for some time interval before returning to the classical system. This latter option is the one we choose as it allows us to fix the system in the false vacuum and then count the number of times it tunnels when it is sent for a given period to $s_{q}$. It is shown beginning as the blue line in Fig. 5, returning to $s=1$ on the orange line.

The value of $s_{q}$ (i.e., the regime where we induce quantum mechanical behavior) is much larger $\left(s_{q}=0.65\right.$ in Fig. 5 ) than would normally be the case. Figure 4 makes it clear that we will choose it to be where quantum mechanics is just turning on in order to have relatively slow tunneling and maintain good control.

During the annealing we choose an $h$-gain schedule, $C(t)$, which varies between $C_{0}<1$ and $C_{0}=1$, as indicated by the green line in Fig. 5. For an initial period the $h$ gain begins at a small enough value such that the second minimum induced by $U_{1}$ is higher than that at the origin $U_{0}$. During this initial relaxation and dissipation period, the system is unable to tunnel, so ultimately it is expected to reach the ground state of $U_{0}$ given by Eq. (4). Once it is in a stable bound state, we can adjust $C(t)$ to send the coupling $k \rightarrow 1$, and turn on tunneling for the rest of the annealing. This configuration, in which we first allow the system to settle, is forced on us by the quantum properties of the annealer. Indeed if we were to start the system at the bottom of the metastable minimum at the origin and then simply turn on the transverse field, the system would tunnel very rapidly. This is because in a reverse annealing the classical starting point is a predefined set of $\sigma_{i}^{Z}$ values. This implies that the initial wave function $\psi(\phi)$ is a position eigenstate (it is essentially a Dirac $\delta$ function), containing superpositions of all energy eigenstates.

It is worth mentioning several moves that are required to improve performance. For all our results we use a minor embedding on the D-Wave Systems annealer quantum processing unit, due its limited connectivity, with $N=200$

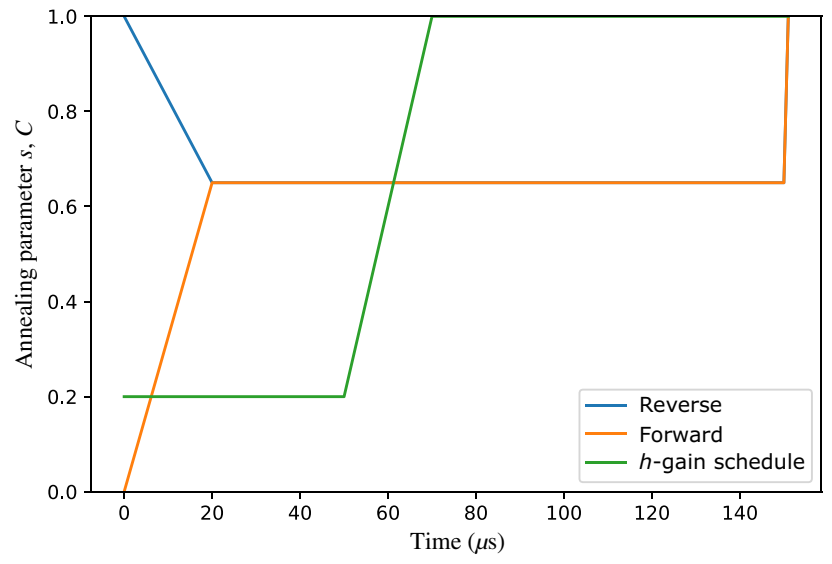

FIG. 5. Typical reverse annealing schedule. The annealing parameter $s$ increases the transverse field, and there is an initial period of stabilization in the minimum at the origin. The $h$-gain parameter is then turned on to introduce metastability and induce tunneling.

qubits in our effective Ising model (but obviously with more on the physical machine due to the embedding). Performance is improved by splitting the large number of reads into smaller groups (of, say, 100) to reduce biasing from each embedding. The states are reinitialized at the bottom of the false vacuum in a classical state at the beginning of each read. As mentioned, one also has to be careful to set the Ising chain parameters, namely, $\Lambda$ and $\Lambda^{\prime}$, to be not much larger than the largest energy scale in the problem. This is because, as mentioned, we wish to avoid the annealer autoscaling the couplings to $\hat{h}$ and $\hat{J}$ as in Eq. (16). After such scaling, Ising chain parameters that were very large would imply couplings in the physical potential that were very small. The effect of autoscaling is actually an additional motivation for our favoring Pöschl-Teller potentials, because they go to a constant at large field values and different $\phi$ intervals do not change the autoscaling: by contrast, a quartic potential would grow rapidly at large field values [32]. Conversely, if the Ising chain parameters are too small, then the Ising chain breaks and we no longer have a faithful representation of the field value. Such "wall breaks" happen a small percentage of the time and can never be eliminated entirely. Those results are simply discarded. Additionally, the minor embedding itself (which ties qubits together in a fashion similar to the Ising chain embedding in $J$ ) may also fail. The parameters can usually be adjusted so that these "chain breaks" happen rarely however.

\section{RESULTS}

\section{A. Calibration on simple-harmonic-oscillator ground states}

We now turn to the results, and discuss the various parameters and further interpretation as we proceed, 


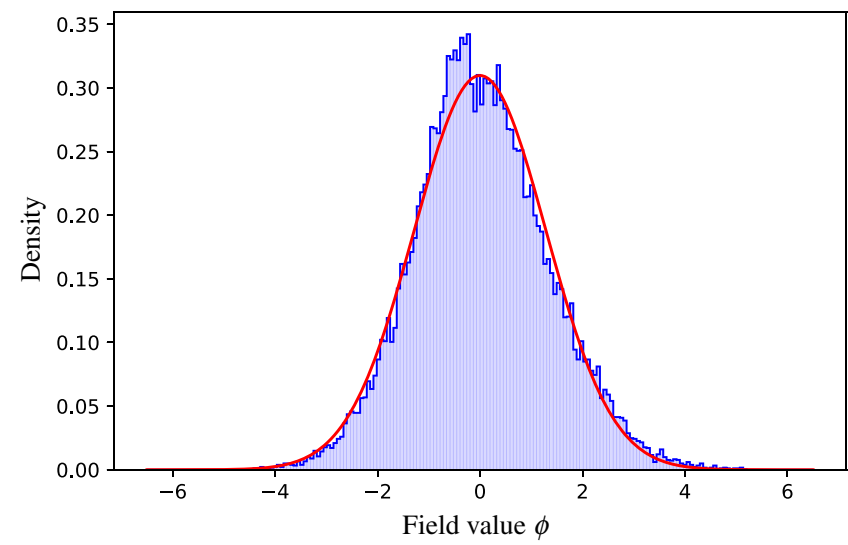

FIG. 6. The probability density of the simple harmonic oscillator with $N=200$ and with $s_{q}=0.7$ after time $t=75 \mu$ s and with $\kappa=0.06$. The ground states are measured with an interval $\Delta \phi=13$. The probability density approximates the red line, which corresponds to $\gamma \equiv \hbar^{2} / 2 m \eta_{0}^{2}=0.33$.

beginning by studying the system with no tunneling. That is, we keep $C(t)=C_{0}$ and set $v$ to be very large in order to learn about the effective Planck constant, more precisely the combination $\gamma=\hbar^{2} / 2 m \eta_{0}^{2}$. As mentioned, this amounts to our calibration of the QASP, and to perform it in a systematic way, we use the simple harmonic oscillator. That is, we take

$$
U_{0}(\phi)=\frac{\kappa}{2} \phi^{2} .
$$

We show the result of 30000 reads of the annealer with $\kappa=0.06$ in Fig. 6, presented as binned probability density functions normalized to 1 . (In other words as $N \rightarrow \infty$, this curve would be $|\psi|^{2}$.) The value of $\kappa$ is chosen small enough to avoid autoscaling. For this run we hold the annealer at $s_{q}=0.7$ for $75 \mu$ s (plus $5 \mu$ s of ramp-up and $1 \mu \mathrm{s}$ of ramp-down).

By inspecting this and similar curves, one gains some intuition about the behavior of this system. First, apart from some seemingly characteristic perturbation around the peak, it clearly appears to have reached the Gaussian ground state, which is of the form

$$
|\psi|^{2}=\frac{(\kappa / 2 \gamma)^{1 / 4}}{\pi^{1 / 2}} e^{-\sqrt{\kappa / 2 \gamma} \phi^{2}},
$$

so we can reasonably conclude that for this choice of parameters $75 \mu \mathrm{s}$ is long enough for the required dissipation. Note that $\eta_{0}$ cancels in the $\kappa / \gamma$ ratio. Second, this curve leads to an approximate estimation of $\gamma=0.33$. Choosing different physical couplings appears to yield similar values of $\gamma$, so not only do the wave functions have the correct shape but they also have the correct functional dependence on $\kappa$. By contrast, the result for the inferred value of $\gamma$ does depend on the interval we choose for $\phi$.

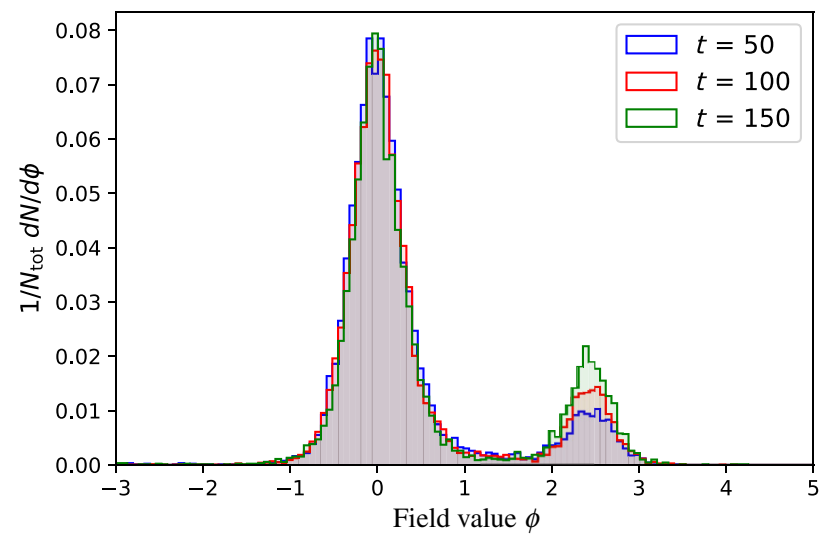

FIG. 7. The probability distribution with $v=2.5$ and $s_{q}=0.7$ after $t_{\text {tunnel }}=50,100$, and $150 \mu \mathrm{s}$, where $N$ is the number of events.

This is because different intervals with the same choice of $N=200$ imply different $\xi$ values, and not surprisingly this affects the mass density $m$ in the field theory.

We stress that absolutely no dynamics is introduced by hand into the annealer, and therefore this constitutes a genuine measurement of the ground-state wave function of a quantum mechanical system.

It is also instructive to consider the fact that the annealer returns a wave function with different $\gamma$ values depending on the value of $s_{q}$. When we choose $s_{q}$, we imbue the effective field theory with a kinetic $\dot{\phi}^{2}$ term that has a certain value of $\hbar^{2} / 2 m$ we do not know. The ground state has to adjust to have the matching value of $\gamma$. Clearly, as we let $s_{q} \rightarrow 1$, the value of $\hbar^{2} / 2 m$ in our effective theory must go to zero because quantum effects turn off there. Accordingly the ground-state wave function becomes increasingly narrow until in the classical limit it approaches a $\delta$ function, which in a reverse annealing is where it begins. In other words the "classical" $\delta$ function position eigenstate

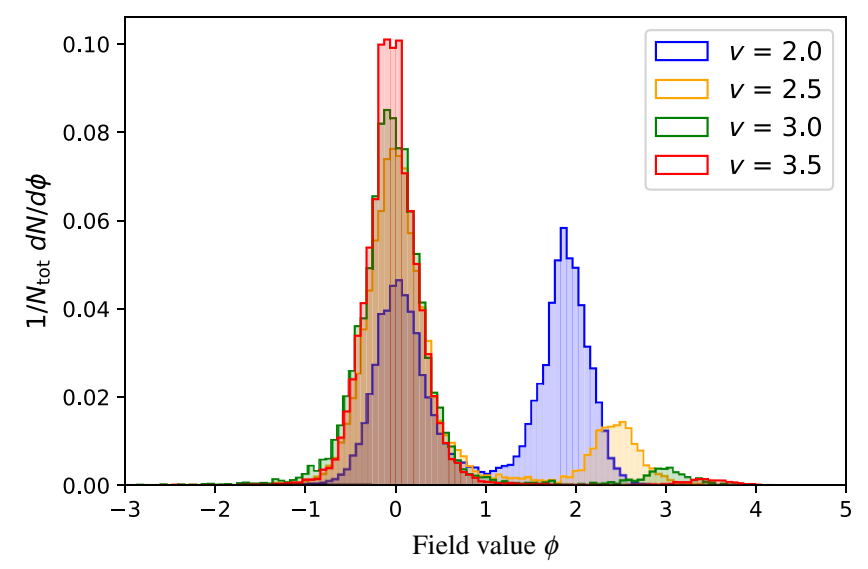

FIG. 8. The transition probabilities for different values of $v$ with $s_{q}=0.7$ after $t_{\text {tunnel }}=100 \mu \mathrm{s}$. 


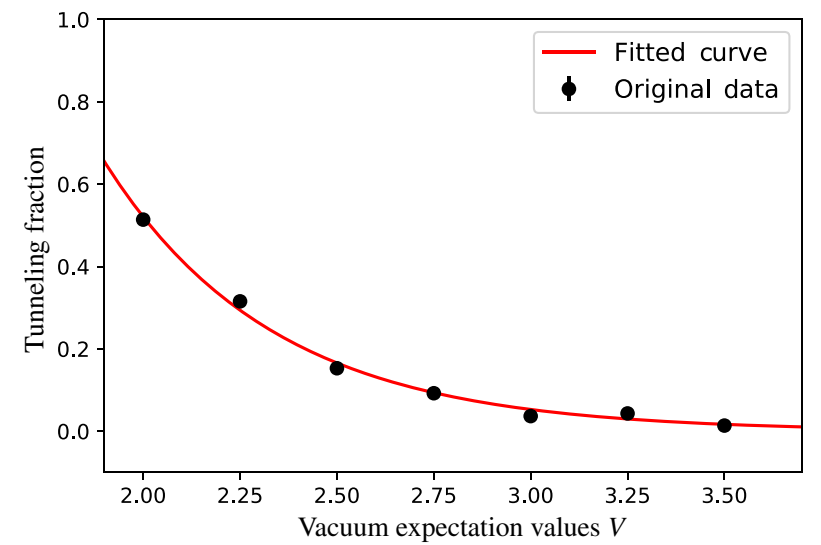

FIG. 9. Best-fit values for the tunneling fraction $P(v)=a e^{-b v}$ for various vacuum expectation values $v$ with tunneling time $t_{\text {tunnel }}=100 \mu \mathrm{s}, a=50.5$, and $b=2.29$.

is simply the ground-state wave function when there is no transverse field component.

\section{B. Tunneling}

We now turn to our double-well potential, and adjust the $h$-gain schedule so that setting the second minimum appears and the system is able to tunnel into it for a period $t_{\text {tunnel }}$. One can perform the same exercise as for the simple-harmonic-oscillator ground state. The result (now displayed as a probability distribution such that the sum of the bin counts is normalized to unity) is shown in Fig. 7 for the system when it is left for 50,100 , and $150 \mu \mathrm{s}$ in the presence of the second minimum, with $k=1$ in the potential of Eq. (1), where we take $v=2.5$. The presence of tunneling is clearly evident. Further evidence in support of this being genuine quantum tunneling can be found by studying the decay rates as a function of $v$. This is shown in Fig. 8 for several values of $v$, where the expected exponential suppression of the decay rate with increasing $v$ is apparent. This exponential behavior can be fit to the approximation in Eq. (14), as in Fig. 9. For the measured value of $\gamma$, the theoretical expectation is $\log \Gamma=3.0 \times(1.66-v)$. The best-fit value (given by the red line in Fig. 9) is $\log \Gamma=2.29 \times(1.71-v)$. Perhaps unsurprisingly, the overall parameter $\gamma$ remains one of the most difficult aspects to determine precisely given the limitations of the annealer for this study. Nevertheless, the observed behavior provides good support for the presence of quantum tunneling.

\section{Quantum versus thermal: study with barriers}

It is important to definitively exclude the possibility that what is being observed is thermal tunneling rather than quantum tunneling. More precisely, we wish to establish that the states are really tunneling through the barrier rather than being thermally excited over the top, noting,

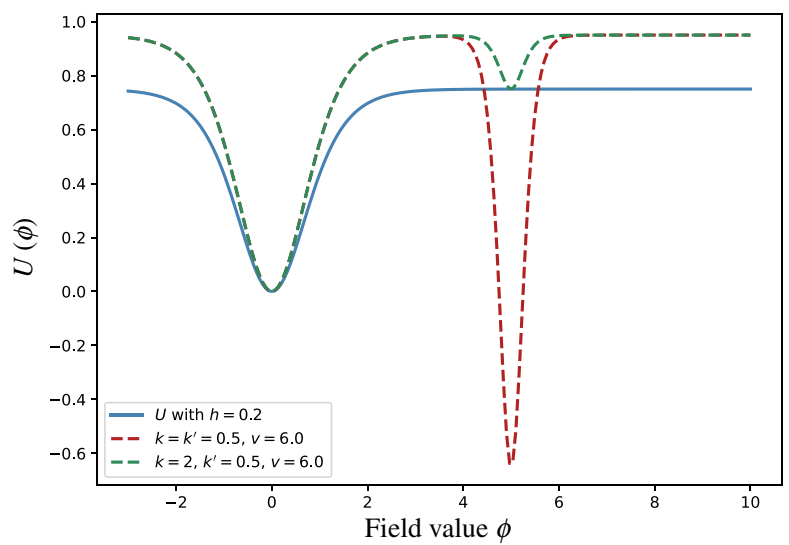

FIG. 10. Minimally disturbing the initial state in order to test if the tunneling exhibits quantum or thermal behavior. The initial potential is a single well, and additional terms raise a barrier between it and a new well that is introduced with a minimum either at either exactly the same height as the original potential or deeper than the original one.

for example, that an explanation for the drop-off with $v$ observed in the tunneling rate above could simply be due to the height of the barrier (and hence the activation energy $E_{a}$ ) increasing with $v$.

To probe this particular question, we now examine a potential that provides a cleaner separation between quantum and thermal behavior, as shown in Fig. 10. The potential is divided up more precisely than before, in the manner described earlier, so that it is of the form in Eq. (23), where we take $C_{0}=0.2$ as our initial $h$-gain parameter. In other words, the terms in our new potential can be written as

$$
\begin{aligned}
& U_{0}=\frac{3}{4} \tanh ^{2} \phi-C_{0} U_{1}, \\
& U_{1}=k^{\prime} \tanh ^{2} \phi-k \operatorname{sech}^{2} c(\phi-v),
\end{aligned}
$$

with the potential at $t=0$ being the single Pöschl-Teller well, shown as the solid blue line. When $C(t) \rightarrow 1$, the first term in $U_{1}$ then raises the sides of the well by $\left(1-C_{0}\right) k^{\prime}$, while the second term introduces a new well at $\phi=v$ of width approximately $1 / c$ and depth $\left(1-C_{0}\right) k$. We take $c=3$ and $k^{\prime}=1 / 2$. We then consider $k=k^{\prime}$ or $k=2$. For this study we also choose $s_{q}=0.65$, which gives more rapid tunneling, allowing us to choose values of $v$ that are in the flat region of the potential.

There are several reasons that this constitutes a clean separation of quantum and thermal behavior. First, it is notable from the study above that the bound state in which the system begins has a rather high energy. Therefore if we simply introduce a new minimum as we did earlier, then it is likely that some components of the wave function will be able to tunnel rapidly. The initial dip at $v$ that is present in our previous configuration would also be able to capture states during the dissipation phase. Neither of these two 
types of state could be very easily distinguished from ones that had thermally tunneled.

What do we expect the tunneling behavior to be in the potential above? In the situation where $k=k^{\prime}$, no new minimum is introduced that would be quantum mechanically accessible to any component of the initial bound state. Therefore, in principle, we should not find any states in this minimum at all if the system is purely quantum, although in practice this will depend on there being no remaining continuous component in the spectrum at all. This is in contrast to the case where $k=2$ shown as the dashed red line in Fig. 10, where the standard quantum tunneling should occur. Moreover, according to Eq. (14), the observed tunneling rate into this minimum should again drop off with increasing $v$, even if we consider values of $v$ in the region where the barrier height is constant.

Let us contrast this behavior with what one would expect for a thermally activated system. In this case there would be little distinction between the $k=1 / 2$ and $k=2$ cases. Once thermal effects are large enough to excite states over the barrier, roughly similar proportions would be captured by the new minimum at $\phi=v$. How much remains trapped there depends somewhat on the temperature and whether the transitions are in equilibrium. If we call the minima at 0 and $v, A$ and $B$, respectively, and the height of the barrier $E_{a}$, ultimately such a system would attempt to reach an equilibrium where the transition rates are the same in both directions; that is, $N_{A} / N_{B}=e^{E_{a} / k_{B} T} e^{-\left(E_{a}-E_{B}\right) / k_{B} T}=$ $e^{E_{B} / k_{B} T}$. If the system were fully in equilibrium, then the ratio of the numbers of states found in the new minima would be independent of the height of the barrier and of order $e^{\left(E_{B_{1}}-E_{B_{2}}\right) / k_{B} T}$, where 1 or 2 labels the choice $k=k^{\prime}$ or $k=2$, respectively. However, the difference in energies $\left(E_{B_{1}}-E_{B_{2}}\right)$ is of the same order as the activation energy $E_{a}$ itself. Therefore a significant thermal tunneling would result in similar numbers of states in the new minima. The

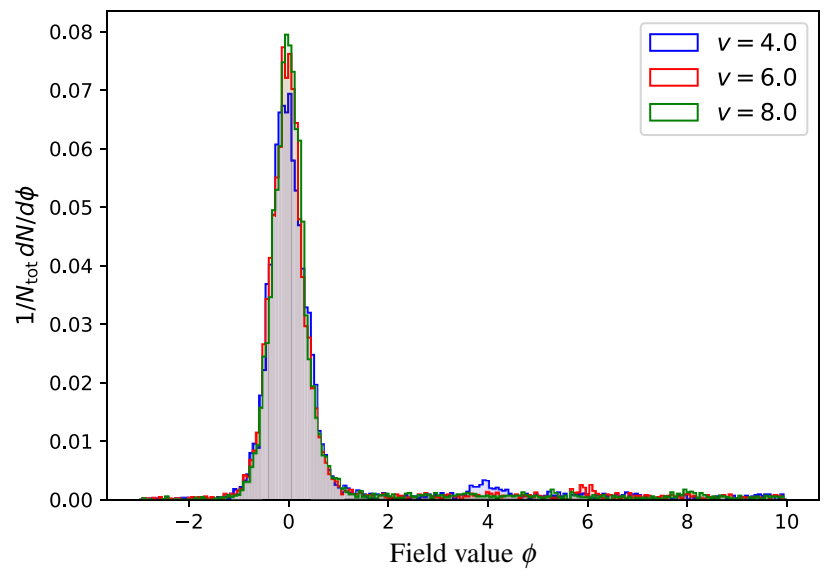

FIG. 11. The transition probabilities into the raised minimum in Fig. 10 for $v=4$ with $s_{q}=0.65$ after $t_{\text {tunnel }}=100 \mu \mathrm{s}$.

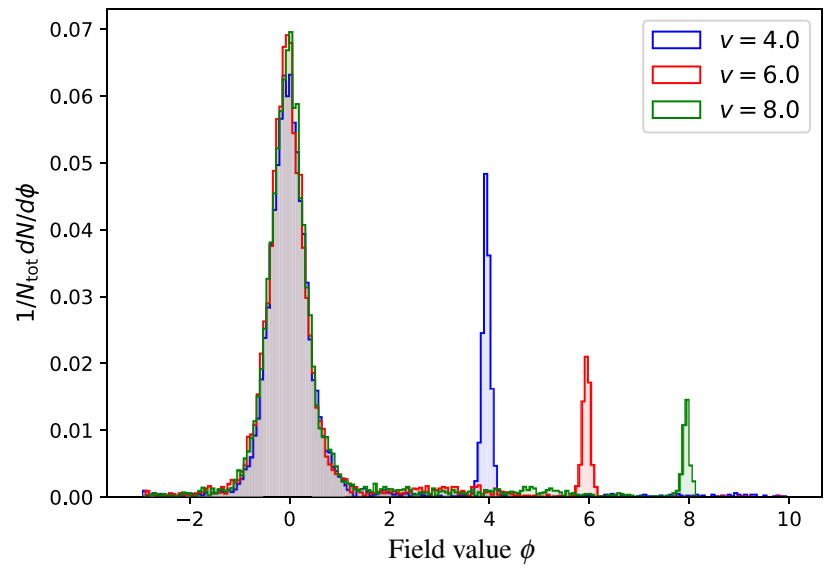

FIG. 12. The transition probabilities for different values of $v$ in the presence of the deep minima in Fig. 10 with $s_{q}=0.65$ after $t_{\text {tunnel }}=100 \mu \mathrm{s}$.

$k=2$ and $k=k^{\prime}$ cases become more similar only if the transitions begin to fall out of equilibrium, as the rate of tunneling in either direction would become very low: the number count in the new minimum would then simply depend on how many states had fallen into its domain of attraction, and this would be virtually independent of the depth. Finally, the tunneling rate should not depend on $v$ in this potential if tunneling proceeds by thermal activation: any thermally activated state would be equally likely to fall into the new minimum regardless of $v$.

Results from the two cases $k=k^{\prime}$ and $k=2$ are shown in Figs. 11 and 12, respectively. The former shows the expected quantum tunneling behavior with a rapid fall in tunneling probability as $v$ increases. The latter has collected some of the energetic degrees of freedom but only a fraction of the number that are able to tunnel into the lower minimum. This behavior provides further support for the presence of quantum tunneling. Essentially we are realizing a many-qubit version of the study of this phenomenon in Refs. [33,34].

There are other simple setups that probe different aspects of the physics, and these will be the subject of future study. For example, one could construct a potential with a small but thin extra barrier in front of the second well. A thermally excited transition would be greatly reduced by such a barrier, while a quantum transition would be virtually unaffected. A point we wish to emphasize however is the ease with which our framework allows one to formulate and address the question.

\section{Quantum versus thermal: no-barrier case}

Unequivocal evidence that the tunneling observed as described above is being driven by entirely quantum behavior can be obtained by studying dynamics in the most trivial case of all - namely, the situation in which there is no barrier. By looking at this case, one can learn 


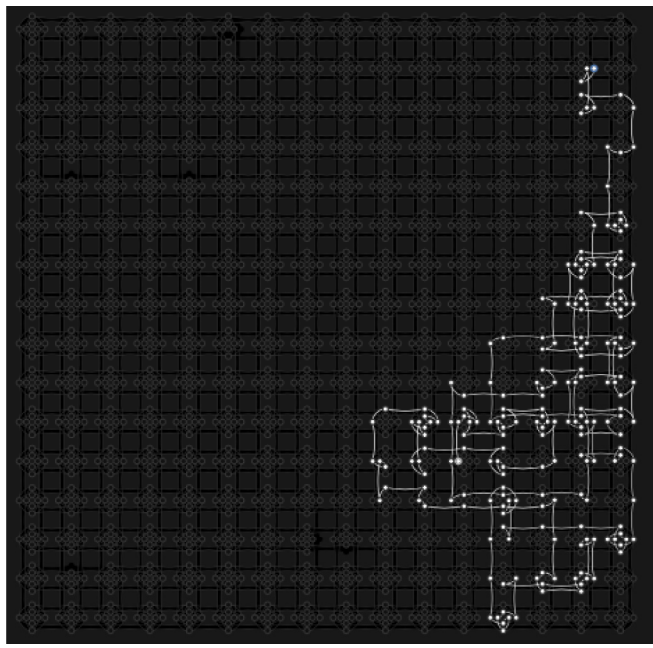

FIG. 13. Typical embedding of the Ising model with $N=200$ on the annealer.

much more about how the field theory dynamics emerges and how it depends crucially on the parameters in the annealer. To this end we consider the system with $U=$ $3 / 4 \tanh ^{2} 3 / 4 \phi$, but beginning with the field displaced at $\phi(0)=-2$.

In such a system one expects the wave function to evolve to $\phi=0$, but the speed with which it does so depends on the nature of the embedding and the parameters we choose. For the present system with its onedimensional encoding, we present a typical embedding on the annealer in Fig. 13. It retains a simple structure, with the position $\phi$ represented by the node positions. To understand the dynamics of the macroscopic field theory we are modeling, it is useful to consider how the system changes in terms of this underlying microscopic structure.

Suppose one starts the system in a reverse annealing with the $\sigma^{Z}$ values chosen initially so as to put $\phi(0)=$ -2 . On an interval $\phi \in[-5,5]$ with $N=200$, this means that 140 of the qubits, in the top half of Fig. 13 say, begin with a $\sigma^{Z}$ eigenvalue of +1 , while the remaining 60 have eigenvalue -1 . As there is no barrier in the potential, one expects the qubits to begin flipping one by one at the domain-wall position, so the domain wall moves to the position corresponding to $\phi=0$, in which exactly half the eigenvalues are positive and half are negative.

However, we observe that this does not happen unless the transverse field contribution is made sufficiently large by dialing down $s$. As an example we show the evolution (or rather lack of it) in Fig. 14. Even though the Ising model in the continuous $N \rightarrow \infty$ limit reproduces the potential $U(\phi)$, one has to recall that the system is actually discrete. For a single qubit in the chain to flip its eigenvalue, even at the site of the domain wall, it evidently has to penetrate a tiny barrier, of order $1 / N$ times the potential. On a microscopic level (by which we mean at the level of

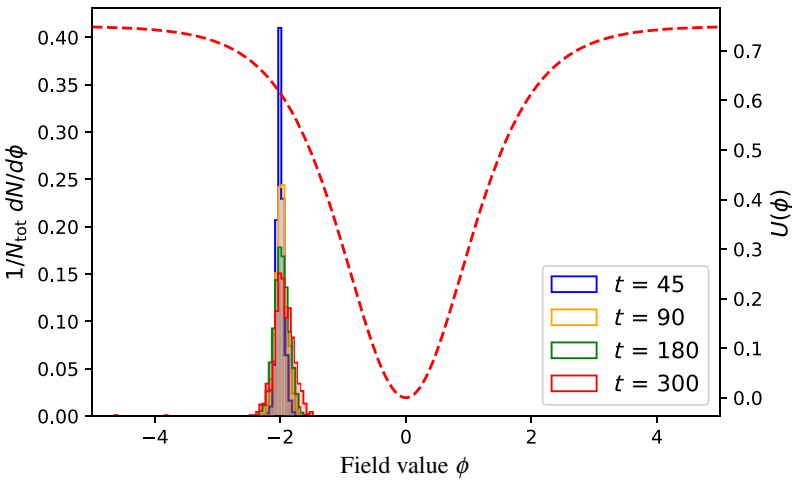

FIG. 14. When $s$ is made large, evolution stops. Here we show the wave function in the near classical regime with $s=0.8$ superimposed on the potential $U=3 / 4 \tanh ^{2} 3 / 4 \phi$.

the discrete Ising model rather than the continuous field theory) the potential is actually a $\tanh ^{2}$ function overlaid with tiny bumps, and the theory has very little dynamics if it cannot penetrate them. Consequently at $s=0.8$ we see very little movement in the domain wall, which remains frustrated at $\phi=-2$.

Dynamics in the macroscopic field theory can emerge once the transverse field term is turned on, allowing the tiny bumps to be penetrated and the domain-wall position to move. This is shown for $s=0.7$ in Fig. 15, where we see the expectation value move to the correct minimum in time $t \approx 50 \mu \mathrm{s}$. What has happened in the effective field theory when $s$ is reduced is that it has acquired a kinetic term and an $\hbar^{2} / 2 m$ term in its effective Schrödinger equation. We conclude that without the transverse field there is no kinetic term in the effective theory, which we can now identify as being a quantum-induced term depending on $A(s)$. Likewise, tunneling in the effective field theory corresponds on a microscopic level to coherent multiple-spin flips, which also turn off exponentially fast with $\gamma$, as in Eq. (13). Crucially this excludes the possibility that the coherent tunneling through large barriers could be anything other than quantum. Finally, it is worth noting the

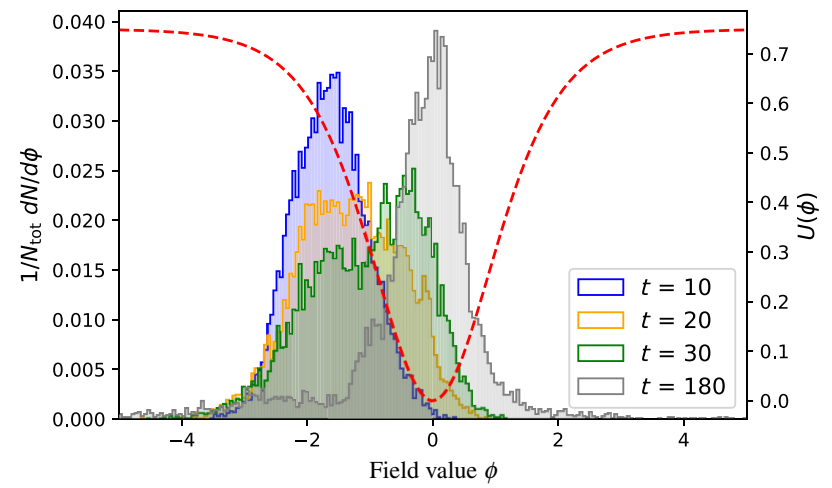

FIG. 15. Evolution in the barrierless potential for $s=0.7$. 


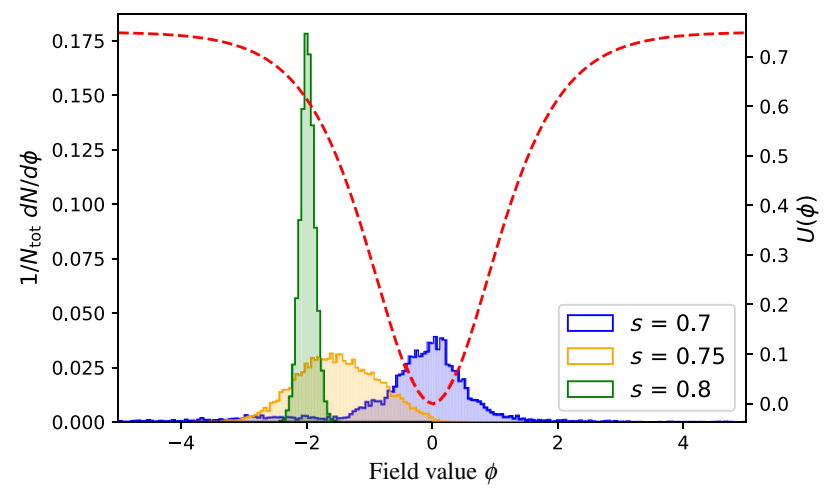

FIG. 16. A snapshot of the wave function after $t=180 \mu \mathrm{s}$ for different values of the annealing parameter $s$.

extreme sensitivity of the effective $\hbar / 2 m$ to the value of $s$. In Fig. 16 we show the wave function after $t=180 \mu \mathrm{s}$ for several values of $s$. Lower values of $s$ correspond to very large $\gamma$ and in the macroscopic system very rapid barrier penetration.

\section{UNIVERSALITY}

The QASP approach appears to be rather flexible and could conceivably be used to probe all kinds of nonerturbative processes in various different field theories. The $d=1$ system (with the single dimension being time) already includes all the paraphernalia of field theory, including loop corrections, particle pair production, and so forth. In principle then it presents a promising simulation platform for testing both perturbative and nonperturbative aspects of quantum field theory.

A natural question to ask is how universal the approach can be. There are three basic augmentations one might consider when evaluating this, which can be broadly classified as additional external symmetries (i.e., space-time degrees of freedom), additional internal symmetries (i.e., gauge degrees of freedom), and different space-time statistics (i.e., fermions).

The first two would be straightforward to implement, with the limitation coming only from the number of available qubits, so only the limited size and connectivity of the annealer prevent us implementing such extensions currently. For example, the number of space-time degrees of freedom could be straightforwardly augmented following the conventional "worldline" approach (reviewed in Ref. [35]). This first-quantized approach would simply require augmenting the number of fields so that $\eta \rightarrow \eta^{\mu}$, with $\mu$ labeling the space dimension. Possibly of more interest is tunneling in the second-quantized formalism, in which one is interested in a field living in a multidimensional space-time. Suppose, for example, one wished to introduce a single space dimension into the field theory as well as the physical time. To do this one would introduce an additional discretization of space into the Ising model as described in Ref. [2]. Namely, we begin by splitting the single space dimension $\rho$ into $M \gg 1$ discrete values and the field value at the $\ell$ th position into $N \gg 1$ discrete values:

$$
\begin{aligned}
\rho_{\ell} & =\ell v=v \ldots M v, \\
\phi\left(\rho_{l}\right) & =\phi_{0}+r \xi=\phi_{0}+\xi \cdots \phi_{0}+N \xi,
\end{aligned}
$$

where $v$ is the unit of discretized space. Now each of the $M$ blocks of qubits holds the field value for that space position:

$$
\phi\left(\rho_{\ell}\right)=\phi_{0}+\frac{\xi}{2} \sum_{j=1}^{N}\left(1-\sigma_{\ell N+j}^{Z}\right) .
$$

Thus our Ising interaction $J_{i j}$ is an $(M N) \times(M N)$ matrix, while $h_{i}$ becomes an (NM) vector. The domain-wall implementation of each field value involves a trivial extension of $J_{i j}^{\text {chain }}$ to the $M$ blocks. We may now introduce kinetic terms into the bilinear $J$ by having interactions that couple adjacent $\ell$ positions. That is, kinetic terms can be discretized in $\rho$ as

$$
\begin{aligned}
\int_{0}^{\Delta \rho} d \rho \frac{1}{2} \phi^{\prime 2}= & \lim _{M \rightarrow \infty} \sum_{\ell=1}^{M} \frac{1}{2 v}\left[\phi\left(\rho_{\ell+1}\right)-\phi\left(\rho_{\ell}\right)\right]^{2}, \\
= & \sum_{\ell=1}^{M-1} \sum_{i j}^{N-1} \frac{\xi^{2}}{8 v}\left(\sigma_{(\ell+1) N+i}^{Z}-\sigma_{\ell N+i}^{Z}\right) \\
& \times\left(\sigma_{(\ell+1) N+j}^{Z}-\sigma_{\ell N+j}^{Z}\right),
\end{aligned}
$$

where $v=\Delta \rho / M$ scales so as to keep $\Delta \rho$ constant. If we insert the discrete representation of the field values using Eq. (27), then Eq. (28) is represented by the following bilinear interactions:

$J_{\ell N+i, m N+j}^{(\mathrm{QFT})}=\frac{\xi^{2}}{8 v}\left(\begin{array}{cccccc}1 & -1 & & & & \\ -1 & 2 & -1 & & & \\ & -1 & 2 & -1 & & \\ & & & \ddots & & \\ & & & -1 & 2 & -1 \\ & & & & -1 & 1\end{array}\right)_{\ell m}$

It was demonstrated in Ref. [2] that this can correctly reproduce classical soliton solutions. The introduction of full Lorentz invariance into the fields encoded on the annealer would then follow straightforwardly, in principle allowing one to recreate instanton processes in conventional relativistic quantum field theories in $d=4$ spacetime dimensions. However the practical limitation here is the number of qubits required, which grows exponentially with the dimensions. A fully fledged $d=4$ theory would 
require $N M^{3}$ qubits: that is, a discretization of a scalar field theory and the space-time it lives in into, say, 50 units would require $6 \times 10^{6}$ qubits.

Additional global symmetries could be encoded rather simply by extending the field content in the obvious manner. The Ising encoding of fermions and of gauge theories is more intricate and a more long-standing topic (see Refs. $[36,37])$. For example, gauge theories would most likely require the encoding of a gauge plaquette action into the Ising model, possibly in the form of dual lattice variables [37,38]. All the usual methods that have been devised for encoding such degrees of freedom in an Ising formalism should be applicable here.

While we are discussing universality, we should mention one caveat, which is that the technique we are using is not expected to be universal in the same general sense that the D-Wave Systems annealer is not in that it is most effective for modeling quantum tunneling processes that occur when a system finds the ground state and then tunnels out of it. It would be more difficult to arrange for large coherent scattering processes (e.g., soliton collisions).

\section{CONCLUSION}

Barrier penetration is a manifestly quantum mechanical property of a quantum field. While such tunneling processes have been observed and studied in quantum mechanics and a selection of special quantum field theories have been realized in nature (e.g., in some condensed matter systems), to our knowledge it has not yet been possible to implement, observe, and study such instanton processes in a freely chosen quantum field theory.

For this purpose we outline how to encode and probe a quantum field theory on a specially prepared QASP. The quantum field is represented by a spin chain and each node corresponds to a qubit on a quantum annealer. After initializing the quantum field with a field value in the potential minimum, one can observe it settle into a quantum eigenstate characteristic of the potential profile imposed on the system. In a second step, we modify the energy profile of the quantum annealer across its qubits, such that the quantum field is no longer in the global potential minimum but is in a false vacuum. We then measure the probability for the field to tunnel from the false vacuum to the true vacuum for various tunneling times, vacuum displacements, and potential profiles. It is then possible to compare the observed tunneling probabilities with those predicted theoretically by the WKB method.

Of course, ultimately the observations in the simpler situations we study here can be reproduced by classical simulations: the result is after all simply a measurement of a solution to the Schrödinger equation, which could always be recovered numerically instead. But it is important to appreciate that this study yields results consistent with a genuine quantum tunneling process occurring on the annealer. A quantum annealer, as, for example, provided by D-Wave Systems, appears to be a platform that, following our method, can be used to encode more general quantum field theories and more complex situations, and probe them physically.

Indeed the exciting prospect is the potential to consider theories and processes that are not easy to treat analytically. As we discuss, the variety of theories that can be studied on a QASP appears to be limited mainly by the number and connectivity of the qubits in the quantum annealer.

This highly adaptive approach could therefore have farreaching implications for future studies of quantum field theories. As measurements of the dynamical behavior of field theories are entirely independent of theoretical calculations, they can be used to infer their properties without being limited by the availability of suitable perturbative or nonperturbative computational methods. The particular dissipative properties of the annealer may also allow the study of decoherence effects in tunneling as discussed, for example, in Refs. [39-41].

Soon, measurements on a QASP could be used to improve theoretical and computational methods conceptually. Furthermore, it could enable the measurement and detailed study of previously unobserved quantum phenomena, involving solitons, instantons, and so forth, that are relevant for field theories of interest in particle physics, condensed matter physics, quantum optics, or cosmology.

\section{ACKNOWLEDGMENTS}

We are grateful to Nick Chancellor for discussions, and to D-Wave Systems and its community for technical support and suggestions during this work.

[1] D-Wave Systems [42] currently provides access to a quantum annealer with 2048 qubits and will provide access a more connected 5000-qubit machine in the future.

[2] S. Abel, N. Chancellor, and M. Spannowsky, Quantum computing for quantum tunnelling, Phys. Rev. D 103, 016008 (2021).

[3] S. R. Coleman, The fate of the false vacuum. 1. Semiclassical theory, Phys. Rev. D 15, 2929 (1977), [Erratum: Phys. Rev. D 16, 1248 (1977)].

[4] C. G. Callan, Jr. and S. R. Coleman, The fate of the false vacuum. 2. First quantum corrections, Phys. Rev. D 16, 1762 (1977).

[5] S. Coleman, Aspects of Symmetry: Selected Erice Lectures (Cambridge University Press, Cambridge, 1988).

[6] I. Affleck, Quantum Statistical Metastability, Phys. Rev. Lett. 46, 388 (1981).

[7] A. D. Linde, Decay of the false vacuum at finite temperature, Nucl. Phys. B 216, 421 (1983), [Erratum: Nucl. Phys. B 223, 544 (1983)]. 
[8] J. Garriga, Instantons for vacuum decay at finite temperature in the thin wall limit, Phys. Rev. D 49, 5497 (1994).

[9] E. Calzetta, A. Roura, and E. Verdaguer, Vacuum decay in quantum field theory, Phys. Rev. D 64, 105008 (2001).

[10] A. Belavin, A. M. Polyakov, A. Schwartz, and Y. Tyupkin, Pseudoparticle solutions of the Yang-Mills equations, Phys. Lett. B 59, 85 (1975).

[11] A. Vainshtein, V. I. Zakharov, V. Novikov, and M. A. Shifman, ABC's of instantons, Sov. Phys. Usp. 25, 195 (1982).

[12] I. Affleck, M. Dine, and N. Seiberg, Supersymmetry Breaking by Instantons, Phys. Rev. Lett. 51, 1026 (1983).

[13] E. Witten, Small instantons in string theory, Nucl. Phys. B 460, 541 (1996).

[14] N. Dorey, T. J. Hollowood, V. V. Khoze, M. P. Mattis, and S. Vandoren, Multi-instanton calculus and the AdS/CFT correspondence in $N=4$ superconformal field theory, Nucl. Phys. B 552, 88 (1999).

[15] J. M. Maldacena, G. W. Moore, and N. Seiberg, D-brane instantons and $K$ theory charges, JHEP 11, 062 (2001).

[16] N. Dorey, T. J. Hollowood, V. V. Khoze, and M. P. Mattis, The calculus of many instantons, Phys. Rep. 371, 231 (2002).

[17] A. Ringwald and F. Schrempp, in Instantons in deep inelastic scattering (2000), p. 318.

[18] G. Brooijmans, P. Schichtel, and M. Spannowsky, Cosmic ray air showers from sphalerons, Phys. Lett. B 761, 213 (2016).

[19] J. Ellis, K. Sakurai, and M. Spannowsky, Search for sphalerons: IceCube vs. LHC, JHEP 5, 085 (2016).

[20] A. Ringwald, K. Sakurai, and B. R. Webber, Limits on electroweak instanton-induced processes with multiple boson production, JHEP 11, 105 (2018).

[21] V. V. Khoze, F. Krauss, and M. Schott, Large effects from small QCD instantons: Making soft bombs at hadron colliders, JHEP 4, 201 (2020).

[22] B. Opanchuk, R. Polkinghorne, O. Fialko, J. Brand, and P. D. Drummond, Quantum simulations of the early universe, Ann. Phys. 525, 866 (2013).

[23] O. Fialko, B. Opanchuk, A. Sidorov, P. Drummond, and J. Brand, Fate of the false vacuum: Towards realization with ultra-cold atoms, EPL 110, 56001 (2015).

[24] O. Fialko, B. Opanchuk, A. I. Sidorov, P. D. Drummond, and J. Brand, The universe on a table top: Engineering quantum decay of a relativistic scalar field from a metastable vacuum, J. Phys. B 50, 024003 (2017).

[25] K. L. Ng, B. Opanchuk, M. Thenabadu, M. Reid, and P. D. Drummond, The fate of the false vacuum: Finite temperature, entropy and topological phase in quantum simulations of the early universe, arXiv:2010.08665.

[26] In nonrelativistic field theories describing spin systems of chiral magnets with a Dzyaloshinskii-Moriya intereaction $[43,44]$ magnetic skyrmions and domain walls have been observed $[45,46]$. Instantons can appear in such systems as composite solitons [47]. Furthermore, the measurement of instantons in special condensed matter systems (see, e.g., Refs. [48-50]) has been reported.

[27] G. Pöschl and E. Teller, Bemerkungen zur quantenmechanik des anharmonischen oszillators, Z. Phys. 83, 143 (1933).
[28] D. Cevik, M. Gadella, S. Kuru, and J. Negro, Resonances and antibound states for the Poeschl-Teller potential: Ladder operators and SUSY partners, Phys. Lett. A 380, 1600 (2016).

[29] E. Brown and L. Pena, A simplified poeschl-teller potential: An instructive exercise for introductory quantum mechanics, J. Chem. Educ. 95, 1989 (2018).

[30] This can be seen using the same techniques [7], but now the finite-temperature field theory is genuinely Euclidean, with compactified time having periodicity given by the temperature-namely, $t_{E}=1 / k T$. The instanton has to satisfy the periodicity condition, and the time coordinate is rescaled accordingly with $\beta=1 / k T$. For high temperatures there is effectively no room for derivative terms in the short interval $\beta$, and we instead find

$$
\Delta_{E} \sim \int_{\phi_{i}}^{\phi_{f}} \mathcal{D} \phi e^{-\beta \int_{0}^{1} d t\left(U-E_{0}\right)} \sim e^{-\beta E_{a}}
$$

with $E_{a}$ being the activation energy to reach the top of the barrier.

[31] N. Chancellor, Domain wall encoding of discrete variables for quantum annealing and QAOA, Quantum Sci. Technol. 4, 045004 (2019).

[32] It is also worth mentioning that the D-Wave Systems annealer does provide the possibility of turning off autoscaling (by setting auto_scale = False) but the performance is reduced unless the couplings are tuned precisely anyway.

[33] S. Boixo, V. Smelyanskiy, A. Shabani, S. Isakov, M. Dykman, V. Denchev, M. Amin, A. Smirnov, M. Mohseni, and H. Neven, Computational role of collective tunneling in a quantum annealer, arXiv:1411.4036 (2015).

[34] S. Boixo, V. Smelyanskiy, A. Shabani, S. Isakov, M. Dykman, V. Denchev, M. Amin, A. Smirnov, M. Mohseni, and H. Neven, Computational multiqubit tunnelling in programmable quantum annealers, Nature 7, 10327 (2016).

[35] J. P. Edwards and C. Schubert, Quantum mechanical path integrals in the first quantised approach to quantum field theory, arXiv:1912.10004 (2019).

[36] J. Alicea, New directions in the pursuit of majorana fermions in solid state systems, Rep. Prog. Phys. 75, 076501 (2012).

[37] J. B. Kogut, An introduction to lattice gauge theory and spin systems, Rev. Mod. Phys. 51, 659 (1979).

[38] C. Gattringer, New developments for dual methods in lattice field theory at non-zero density, PoS LATTICE2013 (2014) 002, arXiv:1401.7788.

[39] T. C. Bachlechner, Decoherence delays false vacuum decay, Classical Quantum Gravity 30, 095012 (2013).

[40] M. Blencowe, Effective Field Theory Approach to Gravitationally Induced Decoherence, Phys. Rev. Lett. 111, 021302 (2013).

[41] N. Bao, A. Chatwin-Davies, J. Pollack, and G. N. Remmen, Cosmological decoherence from thermal gravitons, J. High Energy Phys. 2020, 1 (2020). 
[42] T. Lanting, The D-Wave 2000Q Processor, 2017. Presented at AQC 2017.

[43] I. Dzyaloshinsky, A thermodynamic theory of weak ferromagnetism of antiferromagnetics, J. Phys. Chem. Solids 4, 241 (1958).

[44] T. Moriya, Anisotropic superexchange interaction and weak ferromagnetism, Phys. Rev. 120, 91 (1960).

[45] A. Bogdanov and A. Hubert, Thermodynamically stable magnetic vortex states in magnetic crystals, J. Mag. Mag. Mater. 138, 255 (1994).

[46] U. Roessler, A. Bogdanov, and C. Pfleiderer, Spontaneous skyrmion ground states in magnetic metals, Nature 442, 797 (2006).
[47] M. Hongo, T. Fujimori, T. Misumi, M. Nitta, and N. Sakai, Instantons in chiral magnets, Phys. Rev. B 101, 104417 (2020).

[48] E. Chudnovsky and L. Gunther, Quantum Tunneling of Magnetization in Small Ferromagnetic Particles, Phys. Rev. Lett. 60, 661 (1988).

[49] P. Stamp, Quantum Dynamics and Tunneling of Domain Walls in Ferromagnetic Insulators, Phys. Rev. Lett. 66, 2802 (1991).

[50] X. Zhang, L. Balcells, J. Ruiz, O. Iglesias, J. Tejada, and B. Barbara, Time dependent phenomena at low temperatures in smco multilayers: Quantum nucleation phenomena, Phys. Lett. A 163, 130 (1992). 